\title{
Characterization of factors influencing on-chip complement activation to optimize parallel measurement of antibody and complement proteins on antigen microarrays
}

Running title: Controlling on-chip complement activation

Krisztián Papp ${ }^{\mathrm{a}, \mathrm{d}, 1}$, Péter Végh ${ }^{\mathrm{b}, 1}$, Renáta Hóbor ${ }^{\mathrm{c}}$, Anna Erdei ${ }^{\mathrm{a}, \mathrm{b}}$, József Prechl ${ }^{\mathrm{a}, \mathrm{d}, *}$

${ }^{a}$ Immunology Research Group of the Hungarian Academy of Sciences at Eötvös Loránd University, Budapest, Hungary

${ }^{\mathrm{b}}$ Department of Immunology, Eötvös Loránd University, Budapest, Hungary

${ }^{c}$ Department of Rheumatology and Immunology, Clinic Center, University of Pécs, H7632, Akác utca 1, Pécs, Hungary

${ }^{\mathrm{d}}$ Diagnosticum Zrt, Budapest, Hungary

${ }^{1}$ These authors contributed equally to this work.

*Corresponding author:

Krisztián Papp

${ }^{a}$ Immunology Research Group of the Hungarian Academy of Sciences at Eötvös Loránd University, Budapest, Pázmány Péter s. 1/C

H-1117 Hungary

Tel.: +36-1-2090555 / ext 8664

Fax: +36-1-3812176

e-mail:pkrisz5@gmail.com 


\begin{abstract}
:
Binding of immunoglobulins and complement fragments to targets of adaptive immune responses can be monitored using collections of arrayed antigens and are used to generate profiles of antibody binding and function. The collection of reliable data on these reactions on a large scale requires the establishment of criteria from sample collection through reaction conditions to normalization strategies.

We characterized the detection of $\mathrm{IgG}$, complement $\mathrm{C} 3$ and $\mathrm{C} 4$ under conditions that closely imitate in vivo events and are also relevant for in vitro diagnostic purposes. Immune complex formation was modeled using nitrocellulose-based protein arrays and the effects of factors like anticoagulant use, serum dilution, time and bivalent cation concentrations were assessed. Blood samples from healthy controls $(n=24)$ and patients with systemic autoimmune disease $(n=60)$ were collected and correlations between classical laboratory tests and chip-based reference proteins were evaluated to optimize normalization schemes.

Best signal-to-noise ratio and acceptable masking of IgG by complement $\mathrm{C} 3$ fragments was achieved at modest, five to ten-fold serum dilutions. C3 binding to captured human IgG was found to correlate best with serum C3 concentrations and C3 activity and is therefore an ideal reference feature for normalization of biological and methodological variations in complement activity and detection.
\end{abstract}

Keywords: complement system; C3; IgG

\title{
1. Introduction
}

Immunoglobulins and complement proteins together constitute the second most abundant protein group of the plasma proteome. The complement system is a fundamental element of humoral immunity that constitutes the first protection line against pathogens. It comprises more than 30 proteins, some of which form an orchestrated enzyme cascade with multiple immunological effects. The cascade can be initiated by the classical, lectin or alternative pathways. These routes converge at the point of the cleavage of third complement component $\mathrm{C} 3$ which is followed by the common terminal pathway. Various structures initiate activation of the complement system, e.g. immune complexes (classical pathway); certain carbohydrates (lectin pathway); various microbes, aggregates of immunoglobulin (alternative pathway) (Walport, 2001). The final outcome of the activation depends on the presence of complement control proteins and the properties of initiator, like the isotype, avidity and glycosylation of antibodies in the immune complex (Arnold et al., 2006). Complement pathways can be directly activated by antigen bound antibodies and the efficiency of activation is not predictable undoubtedly knowing their isotypes and or subtypes (Daha et al., 2011), especially when enormous amount of antibody with different properties compete for the same antigen. Activation of the complement system initiates various biological events including cell lysis, inflammation, cell activation and opsonization; these versatile roles emphasize the need for the comprehensive understanding of complement function. Several tests have been developed for analyzing these diverse functions of complement (Mollnes et al., 2007). Most of these techniques measure the amount of complement components or test the three pathways for their intactness (Seelen et al., 2005) but are inappropriate for measuring antigen specific complement activation. 
Immunoglobulins are the most diverse constituents of the plasma proteome. Arrayed antigens can be used to analyze a part of this diversity by generating binding patterns called antibody profiles (Prechl et al., 2010). Interaction between antigen and antibody results in the formation of immune complexes, which further interact with other serum components, such as complement, and cellular receptors. In order to generate a realistic picture about the events ensuing antigen recognition by antibody, we developed a protein microarray-based technique (Papp et al., 2007). On-chip complement activation generates functional antibody profiles and helps characterize immunity in a systematic way (Papp et al., 2008a; Papp et al., 2008b). Following treatment of arrayed materials with slightly diluted serum, we can detect the amount of bound $\mathrm{IgG}$ and complement breakdown products, like C3b, iC 3b, C3d, C4b and C4c fragments, in parallel. Since we did not attempt to determine the degree of fragmentation and also for the sake of simplicity from here on we refer to the various deposited fragments as C3 and C4. Here we report the modifying effect of different reaction conditions, like the type and concentration of blood sample, level of ions and the length of serum treatment, on IgG binding and complement activation in a planar microarray assay. Additionally we identify a normalization strategy that compensates for individual variations of complement activity. Applying suitable conditions during detection of antibodies on microarray, also their important effector function, their complement activating properties is measureable driving us closer to the understanding in vivo pathological processes.

\section{Materials and Methods:}

\subsection{Materials:}

Properdin was purchased from CalBiochem and $\mathrm{F}(\mathrm{ab})_{2}$ fragment of goat anti-human Ig $\gamma$ chain specific antibody (anti-human IgG) from Jackson ImmunoResearch Laboratories. All other materials were purchased from Sigma-Aldrich.

\subsection{Blood collection:}

The study was approved by the national Scientific and Research Ethics Board; informed consent was obtained from each participant. Control serum samples were selected from the repository of the Drug Research Center. Patients with systemic lupus erythematosus (SLE) fulfilled the international criteria. Venous blood from some healthy volunteers was collected in different types of tubes: serum (BD Vacutainer, Plus Blood Collection Tube); EDTA-plasma (BD Vacutainer, K3E Tube, $0.084 \mathrm{ml} 15 \% \mathrm{~K} 3 \mathrm{E}$ in $7 \mathrm{ml}$ tube); heparinplasma (Vacuette Greiner bio-one, lithium heparin containing tube); hirudin-plasma $(50 \mu \mathrm{l}$ hirudin $(50 \mathrm{U})+950 \mu \mathrm{l}$ blood); citrate-plasma (1 $\mathrm{ml} \mathrm{3.8 \%} \mathrm{Na-citrate}+9 \mathrm{ml}$ blood). Plasma was centrifuged at $1000 \mathrm{~g}$ for 10 minutes directly following blood collection; while for serum samples centrifugation was carried out 1 hour later, using the clotted blood. Supernatants (plasma/serum) were divided into aliquots and kept at $-70^{\circ} \mathrm{C}$ until use. Only serum samples with available $\mathrm{C} 3$ concentration data and $\mathrm{CH} 50$ values, as determined by certified clinical laboratories, were included in this analysis.

\subsection{Microarray production and measurement}

Experiments were carried out on nitrocellulose coated 16 pad FAST Slides (GE Healthcare) for the comparison of different reaction conditions and on 2 pad slides for serum profiling. Anti-human IgG, human IgG, properdin, pG and BSA were spotted in triplicates of $1 / 5$ serial dilution (low, medium and high concentration) by BioOdyssey Calligrapher MiniArrayer (Bio-Rad). The starting concentration was usually $0.5 \mathrm{mg} / \mathrm{ml}$ 
except anti-human $\mathrm{IgG}$ and BSA that were used in $1 \mathrm{mg} / \mathrm{ml}$. Ready slides were kept in sealed non-transparent bags at $4^{\circ} \mathrm{C}$.

The basic protocol - that was modified sometimes, as described in the result section - is the following: dried arrays were rehydrated in PBS (4x5min) before using, then subarrays were incubated in $70 \mu \mathrm{l}$ diluted or undiluted sample at $37^{\circ} \mathrm{C}$ for $1 \mathrm{~h}$, providing suitable conditions for complement activation. Sample dilution was carried out usually in Ca-Mg-VBS (2.5mM Ca ${ }^{2+}, 0.7 \mathrm{mM} \mathrm{Mg}^{2+}, 0.05 \%$ Tween $20,5 \%$ BSA, Veronal buffered saline) or when the complement blocking was the goal, ions were replaced with $25 \mathrm{mM}$ EDTA. Plasma/serum treated slides were washed in $0.05 \%$ Tween containing PBS, then incubated at room temperature for 30 minutes with fluorescently labeled detecting antibodies that were diluted in blocking buffer $(0.05 \%$ Tween $20,5 \%$ BSA, $0.05 \%$ azide, PBS). As mentioned in the results section, differently diluted, various antibodies were used for detection: goat $\mathrm{F}(\mathrm{ab})_{2}$ fragment to human $\mathrm{C} 3$ antibody (Cappel, MP Biomedicals) labeled with Alexa 647 dye by us (5000x); ; FITC labeled goat anti-human C4 antibody (Cappel, MP Biomedicals) (2500x); biotinilated F(ab') $)_{2}$ fragment of goat anti-human IgG $\gamma$ chain specific antibody (Southern Biotechnologies) (5000x); PE-Cy5.5 conjugated Streptavidin (eBioscience) (4000x). Antibodies that were used only in experiment that tests the effect of serum dilution: mixture of rabbit anti-human $\mathrm{C} 3 \mathrm{~d}+$ C3c-FITC (Dako) (2500x); allophycocyanin-conjugated $\left.\mathrm{F}(\mathrm{ab})_{2}\right)_{2}$ fragment of goat antihuman IgG $\gamma$ chain specific antibody (Jackson ImmunoResearch Laboratories) (2500x). Then chips were washed again and following drying, slides were scanned on Typhoon Trio+ imager (Amersham Biosciences).

The serum profiling data discussed in this publication have been deposited in the National Center for Biotechnology Information's Gene Expression Omnibus (GEO) (Edgar et al., 2002) and are accessible through GEO series accession no. GSE26768 (www.ncbi.nlm.nih.gov/geo/query/acc.cgi?acc).

2.4 Analysis of microarray data

Images of slides were analyzed with GenePix Pro 6.0 (Molecular Devices, Corp.) software after visual inspection. Relative intensity of fluorescence (RFI= FI median (pixels of spot) -FI median (pixels of local background)) was calculated for each spot, then the mean of the replicates were used for further analysis. Signal intensities were calculated by subtracting background from medians of three parallel signal intensities in a spreadsheet program (Microsoft Excel). Signals not exceeding two standard deviations of local background signals on a slide were clamped to an arbitrary value.

\section{Results}

\subsection{Effect of anticoagulants on complement measurement}

Anticoagulants not only block blood clotting but some of them alter the efficiency of complement activation, as well. We tested the modifying effect of different blood collection methods on on-chip complement detection. Three probes with different obligate complement activating properties were printed on arrays: $F(a b)_{2}$ fragment of anti-human IgG, human IgG and properdin. Plasma containing generally used anticoagulants (EDTA, citrate, heparin, hirudin) was compared with serum, regarding their applicability for array-based complement measurement. Of note, during array treatment the thawed hirudin plasma formed a loose gel on the array surface, even though a recommended, published (Manderson et al., 2001) hirudin concentration was used. The 
amount of bound $\mathrm{C} 3$ and $\mathrm{C} 4$ fragments was assessed following treatment of printed probes with undiluted and 5-fold diluted plasma/serum. The highest amount of C3 fragment was detected in heparin plasma, while the lowest in EDTA containing plasma (Figure 1A). Depending on the probes, the amount of deposited C4 fragments in undiluted plasma/serum was high when citrate and also substantial where EDTA or heparin was used as anticoagulant, but in the case of hirudin plasma and serum, C4 signal was low (Figure 1B). C4 deposition is not expected on properdin spot because it is an alternative pathway initiator. When blood samples were diluted 5-fold in a buffer containing $\mathrm{Ca}^{2+}$ and $\mathrm{Mg}^{2+}$ ions, both $\mathrm{C} 3$ and $\mathrm{C} 4$ signals increased and differences between the blood collecting methods nearly disappeared.

3.2 Correlation between serum dilution and complement activation measurement As it was shown above, dilution of samples increased the signal-noise ratio. In the next step, the effect of serum dilution on antibody and C3 detection was determined. Arrays were incubated in serially diluted serum for 1 hour and the bound IgG and C3 fragments were detected. Images of the slides on Figure 2A show that the higher the dilution the lower the background was. Above 10-fold dilution the $\mathrm{C} 3$ signal decreased on all three complement activators (Figure 2B). In contrast, the IgG signal increased with dilution. That complement activation had a negative impact on IgG detection best showed up on the printed human IgG spots which gave higher IgG signal when the array was incubated with buffer, instead of diluted serum. Anti-properdin antibodies are normally not present in serum explaining the lack of IgG signals on properdin spots.

\subsection{Dependence of complement activation on ion concentration}

Complement activity requires calcium and magnesium ions. $\mathrm{Ca}^{2+}$ ions take part in the recognition and activation phase of classical and lectin pathways and are critical for the stabilization of $\mathrm{C} 1$ and mannose binding lectin complexes (Thielens et al., 2001; Roumenina et al., 2005). $\mathrm{Mg}^{2+}$ ions facilitate factor B binding to $\mathrm{C} 3\left(\mathrm{H}_{2} \mathrm{O}\right)$ or $\mathrm{C} 3 \mathrm{~b}$, and so contribute to the proper working of the alternative pathway. The physiological concentrations of $\mathrm{Ca}^{2+}$ and $\mathrm{Mg}^{2+}$ ions are $2.5 \mathrm{mM}$ and $0.7 \mathrm{mM}$, respectively (WACKER et al., 1965). We tested the effect of five-times lower and higher ion concentrations on complement activation and $\mathrm{IgG}$ detection. Figure 3 shows that deposition of C3 fragments on printed antibodies diminished when 5-times higher than physiological $\mathrm{Ca}^{2+}$ ions were present in the solution. This effect of $\mathrm{Ca}^{2+}$ was not observed with properdininduced activation. On anti-human IgG and human IgG spots $\mathrm{Mg}^{2+}$ ion concentration was positively and negatively related with $\mathrm{C} 3$ and $\mathrm{C} 4$ fragment deposition, respectively. The IgG signals changed inversely with $\mathrm{C} 3$ signals. On properdin spots neither $\mathrm{C} 4$ nor IgG signal was detected.

\subsection{Kinetics of $C 3$ and $C 4$ fragment deposition}

The changes of $\mathrm{C} 3$ and $\mathrm{C} 4$ signals were measured for different lengths of incubation time using the serum of four healthy volunteers. The shapes of the curves were similar for the different serum samples (Figure 4). On the properdin and anti-human IgG spots the C3 signal kept increasing in time, while detectable $\mathrm{C} 4$ signal reached its plateau on antihuman IgG spot already in 10 minutes. On printed human IgG spots C3 levels reached a close to maximum value in about 10 minutes after a very fast initial increase, the $\mathrm{C} 4$ signal reached its peak in 5 minutes, and started decreasing for the next 50 minutes. 3.5 Correlation between total hemolytic activity, $C 3$ concentrations and C3 deposition 
Complement C3 concentrations and hemolytic activity vary between individuals on a relatively wide scale. This is coupled with variations introduced by sample collection, reaction conditions and signal detection steps. To compensate for these effects and in order to generate antigen specific complement $\mathrm{C} 3$ deposition profile that reflect immune responses rather than complement function, datasets need to be normalized. An ideal reaction for this purpose comprises antibody initiated complement activation, independent of serum antibody concentrations, reflects complement $\mathrm{C} 3$ concentrations and activity in a wide range. The complement activating agents characterized above were tested in this respect by correlating $\mathrm{C} 3$ deposition values with serum total $\mathrm{C} 3$ that was measured by a sandwich antibody pair on the array or by nephelometry in a certified laboratory. Correlation with total hemolytic activity $\mathrm{CH} 50$ was also addressed (Figure 5). We used sera from patients with systemic autoimmune disease and matched control subjects to obtain complement activity variations relevant for diagnostic applications (GEO ID: GSE26768). We found that anti-human IgG capture showed consistent positive correlation with total serum C3 and also with hemolytic activity. Printed human IgG showed correlation with chip-based C3 concentrations only. Unexpectedly, properdininduced complement activation showed no or negative correlation with $\mathrm{C} 3$ content or activity, due to the segregation of patients with systemic autoimmune disease from controls (Figure 5.).

\section{Discussion:}

Contact between antigen and blood results in the binding of various recognition molecules to the antigen. Immersing microarrayed antigens in blood results in the generation of binding profiles of these recognition molecules, of which immunoglobulins are most important. It is also possible to follow events triggered by Ig binding, such as complement activation. Parallel measurement of these two reactions provides a functional map of the "immunome". On-chip complement activation is suitable for the parallel measurement of bound antibody and antigen specific complement activation (Papp et al., 2007). Importantly, reproducible and comparable measurement of complement activation requires controlled sample collection, storage, application and normalization conditions. Anticoagulants can interfere with complement function, depending on their mode of action. We compared various blood collection methods for determining their adaptability to the protein array technique. EDTA and citrate block blood coagulation by chelating $\mathrm{Ca}^{2+}$ and $\mathrm{Mg}^{2+}$ ions, which are important for complement activation. Hirudin is a thrombin specific inhibitor that does not interfere with complement (Chang, 1983; Mollnes et al., 2002); the anticoagulant effect of heparin is based on its binding to antitrombin III. The influence of heparin on the complement system is very complex; it has major effect on the alternative pathway but it also interferes with the classical pathway (Weiler and Linhardt, 1989; Weiler et al., 1992). The effect of heparin on complement activation is unusual because it inhibits complement activation at high concentration, whereas enhances the activation at low concentration (Mollnes et al., 2002). When using undiluted plasma/serum, anticoagulants strongly influenced both $C 3$ and C4 fragment deposition (Figure 1). Compared to hirudin, an anticoagulant with no effects on complement, sodium citrate, EDTA and heparin inhibited or enhanced complement deposition. These effects were too complex to be explained by simple interactions and clearly indicated that undiluted plasma containing different 
anticoagulants is not suitable for analysis. Fivefold dilution of the plasma samples in a buffer that contained $\mathrm{Ca}^{2+}$ and $\mathrm{Mg}^{2+}$ ions blunted anticoagulant effects, partly by restoring ions and partly by diluting the agents themselves. Thus, in addition to serum, which is the preferred substrate for testing, hirudinated plasma is also acceptable, while other anticoagulants should be avoided for on-chip complement tests.

Unexpectedly, modest $\mathrm{C} 4$ signal was also observed on the alternative pathway activator properdin spots in the presence of heparin. This was probably the result of antibodies binding to properdin under these conditions (data not shown).

As fivefold dilution of the samples in a buffer containing $\mathrm{Ca}^{2+}$ and $\mathrm{Mg}^{2+}$ ions had dramatic effect on the results, in the next step we tested the dependence of complement deposition and Ig detection on the extent of serum dilution. A relatively strong background $\mathrm{C} 3$ deposition was observed in undiluted serum that decreased gradually with dilution (Figure 2A). This background is probably the result of the continuous alternative pathway tick-over that gives rise to reactive $\mathrm{C} 3$ fragments, which bind to the surface. Best signal-to-noise ratio for $\mathrm{C} 3$ was achieved in the range of 5 to 10 -fold serum dilution, confirming that efficient complement activity requires close-to normal concentrations of complement components. On the contrary, signals of both captured and printed IgG increased as a function of serum dilution (Figure 2B). In fact, original signal strength of the printed IgG was regained only when complement C3 deposition was negligible. These results suggest that deposited C 3 fragments mask the bound antibodies and hinder detection at lower dilutions. Harboe et al. reported that hemolytic activity is lost at 16fold dilution by the alternative and at 1024-fold dilution by the classical pathway (Harboe et al., 2004). Our data is in agreement with those data. Spotted human IgG activates mainly the classical, while properdin mainly the alternative pathway. Printed goat antihuman IgG $\mathrm{F}(\mathrm{ab})_{2}$ fragments also directly activate the human alternative complement system (Reid, 1971); that effect decreases dramatically above the 10-fold dilution which explains the shape of the observed curve.

We tested the effect of bivalent cation concentrations on the three printed test materials. Printed anti-human IgG and human IgG activate the classical pathway that needs $\mathrm{Ca}^{2+}$ ions. As figure 3 shows, 5-fold increase in $\mathrm{Ca}^{2+}$ ion concentrations diminished $\mathrm{C} 3$ fragment deposition. On these spots the binding of $\mathrm{C} 3 \mathrm{~b}$ fragments also initiated the alternative pathway and increasing amounts of $\mathrm{Mg}^{2+}$ ions modestly increased the $\mathrm{C} 3$ signal. Excess of $\mathrm{Mg}^{2+}$ ions overdrives the positive feedback loop of the alternative pathway. Therefore the newly formed $\mathrm{C} 3 \mathrm{~b}$ fragments masked the bound $\mathrm{C} 4$ and $\mathrm{IgG}$ molecules lowering $\mathrm{C} 4$ and IgG signals. Even though the alternative pathway activated by properdin is $\mathrm{Mg}^{2+}$ ion-dependent, reducing $\mathrm{Mg}^{2+}$ concentrations to $20 \%$ of the physiological levels did not decrease the $\mathrm{C} 3$ signal substantially, except in the presence of elevated amounts of $\mathrm{Ca}^{2+}$. Healthy serum does not contain anti-properdin antibodies and lacks IgG and $\mathrm{C} 4$ signals on properdin.

For determining the kinetics of complement fragment deposition, $\mathrm{C} 3$ and $\mathrm{C} 4$ was detected simultaneously at different points of time. C3 signals increased throughout the 2-hour test in all three assays, though at different rates. On printed anti-human IgG spots the $\mathrm{C} 4$ signal reached its plateau at 10 minutes, while on printed human IgG spots, where classical pathway initiates complement activation, $\mathrm{C} 4$ signals increased for 5 minutes, then dropped back to a relatively constant level by 30 minutes. The classical pathway initiator $\mathrm{Clq}$ directly bound to printed human IgG antibodies, explaining the very fast $\mathrm{C} 4$ 
deposition. The $\mathrm{C} 4$ signal decrease that followed is explainable by $\mathrm{C} 4$ degradation and the masking effect of $\mathrm{C} 3$. Overall, complement deposition can be reliably measured starting from 30 minutes, and steady state changes further increase C3 signals for at least another 90 minutes.

Based on these observations complement IgG binding and complement $\mathrm{C} 3$ and $\mathrm{C} 4$ deposition was measured from 5-fold diluted sera, allowing 60 minutes in the presence of physiological cation concentrations for the reaction to take place. Systemic lupus erythematosus is an immune complex mediated disease that is often accompanied by decreased complement activity due to consumption. Complement C3 deposition in serum samples from patients with systemic autoimmune disease and control subjects showed similar correlations to total C3 levels and hemolytic activity as assessed by anti-human IgG capture. Notwithstanding, C3 deposition on properdin was rather independent of total $\mathrm{C} 3$ and $\mathrm{C} 3$ activity levels in patients with systemic lupus erythematosus. Capturing $\mathrm{IgG}$ from serum therefore seems to be suitable for normalizing biological and methodological variations when assaying C3 deposition on protein arrays. In addition to secondary antibodies, such as the goat anti-human IgG used in these assays, B-cell superantigens, e.g. protein $\mathrm{G}$, perform similarly and are potential alternatives (data not shown).

Trouw et al. presented an ELISA based technique for measurement of complement activation of antigen bound antibodies, treating antigen coated wells with EDTA containing patient serum then using a normal human serum as common complement source for all sample (Trouw et al., 2009). This approach also achievable with our microarray method, but based on our experiments, this setup increases the background and the variance of data. Various serum components from active complement containing normal human serum can modify the result, as their react with bound elements of serum samples derived from patient. We suggest applying the serum of patient as complement source as in this way we could gain information really the serum reactivity of the given individual. Using suitable control spots on the same array, differences in overall complement activation is removable, that approach would not be achievable in ELISA method.

In conclusion, these results identify optimal conditions for the parallel measurement of IgG binding and complement activation on protein microarrays. The identified conditions - with limited serum dilution compared other techniques for antibody detection- enables even to the low affinity antibodies to compete for epitopes that better mimic the environment of antigen recognition in blood. With the utilization of IgG capture agents one can normalize $\mathrm{C} 3$ deposition values to obtain datasets that reflect complex interactions between antigen panels and the serum proteome.

\section{Acknowledgements}

Research on antigen microarrays is supported by the Hungarian Academy of Sciences, KMOP 1.1.1-08/1-2008-28 grant by the National Agency for Development, OTKA K72026 of the Hungarian Scientific Research Fund and Cell-Kom RET 2006/06. Krisztián Papp supported by János Bolyai Research Fellowship. We wish to thank Tímea Berki (University of Pécs) and Julianna Németh (ANTSZ Laboratory, Budapest) for their help with clinical diagnostic laboratory tests. The authors thank Prof. László Czirják 
(University of Pécs) for his advice on study design and for critically reading the manuscript.

\section{Conflict of interest}

K.P. and J.P. are currently employed by Diagnosticum Zrt, the company that licenses rights of a patent application on complement activation assays on microarrays. The owner of the application is Eötvös Loránd University and Hungarian Academy of Sciences. 


\section{Figure legends}

Figure 1.: Effect of anticoagulants on on-chip complement activation

Proteins that initiate complement activation were printed on nitrocellulose as arrays. Goat anti-human $\operatorname{IgG}$ captures IgG from serum, while printed human IgG directly triggers classical pathway activation. Properdin stabilizes $\mathrm{C} 3$ convertase of the alternative pathway. The arrays were treated with plasma containing the indicated anticoagulants or serum, each undiluted or in a 1:5 dilution. The amounts of bound C3 (A) and C4 (B) fragments were detected using fluorescently labeled secondary antibodies. Blood samples with different anticogulants were collected at the same time from three different healthy donors and each experiment was performed twice. Relative fluorescence intensities (RFI) are shown.

Figure 2.: Serum dilution influences protein detection For the comparison of reaction conditions, 16-pad arrays were incubated with serially diluted serum. FITC conjugated anti-human C $3 \mathrm{~d}+\mathrm{c}$ and anti-human-IgG-APC antibodies were used for detection of bound $\mathrm{IgG}$ and $\mathrm{C} 3$ fragments, respectively. In a control subarray, only buffer was used for incubation. A) Representative image of a chip from four experiments and the layout of the sub-array. B) Average relative fluorescence intensities of the medium diluted printed probes are plotted as a function of serum dilution; SD values are indicated. BB refers to Bromophenol Blue containing spots those printed only for proper positioning.

Figure 3.: Bivalent cations strictly control complement activity Protein arrays were incubated in five-fold diluted serum. Ca-Mg-VBS buffer was used for dilution (see Materials and Methods), with the ion concentrations adjusted to reach the final concentrations shown on the $\mathrm{x}$ and $\mathrm{y}$ axes. Graphs show $\mathrm{C} 3, \mathrm{C} 4$ and IgG RFI values of the three complement activating proteins. Data are representative of four independent experiments.

Figure 4.: Kinetics of complement signals:

Arrays were incubated for different periods of time shown in 5-fold diluted serum, derived from 4 healthy people. The control sub-array, indicated as 0 point, was incubated for $120 \mathrm{~min}$ in $25 \mathrm{mM}$ EDTA containing diluted serum to completely block complement activation. Anti-human C3-Alexa 647 and anti-human C4-FITC detecting antibodies were used for measuring bound C3 (black line, full symbol) and C4 (gray line, empty symbol) fragments on the three complement activators.

Figure 5.: Correlation between on-chip complement $\mathrm{C} 3$ deposition on the obligate activators and total $\mathrm{C} 3$ concentration and activity

C3 deposition was measured in sera from patients with autoimmune disease (Systemic lupus erythematosus, SLE) and control subjects using the complement activating substances described above. Serum complement $\mathrm{C} 3$ was estimated by an antibody pair on the array (anti-human C3) or by nephelometry in a clinical laboratory. Hemolytic activity (CH50 value) was measured by an in vitro diagnostic assay that detects soluble terminal pathway complex C5b-9. Correlations between C3 deposition values and C3 concentration and hemolytic activity are shown. 
Correlation coefficients and $\mathrm{p}$ values of Spearman rank correlations are indicated.

\section{Abbreviations:}

RFI, relative fluorescence intensity;

\section{Reference List}

Arnold, J.N., Dwek, R.A., Rudd, P.M. and Sim, R.B., 2006, Mannan binding lectin and its interaction with immunoglobulins in health and in disease. Immunol Lett 106, 103-10.

Chang, J.Y., 1983, The functional domain of hirudin, a thrombin-specific inhibitor. FEBS Lett 164, 307-13.

Daha, N.A., Banda, N.K., Roos, A., Beurskens, F.J., Bakker, J.M., Daha, M.R. and Trouw, L.A., 2011, Complement activation by (auto-) antibodies. Mol Immunol 48, 1656-65.

Edgar, R., Domrachev, M. and Lash, A.E., 2002, Gene Expression Omnibus: NCBI gene expression and hybridization array data repository. Nucleic Acids Res 30, 207-10.

Harboe, M., Ulvund, G., Vien, L., Fung, M. and Mollnes, T.E., 2004, The quantitative role of alternative pathway amplification in classical pathway induced terminal complement activation. Clin Exp Immunol 138, 439-46.

Mollnes, T.E., Brekke, O.L., Fung, M., Fure, H., Christiansen, D., Bergseth, G., Videm, V., Lappegård, K.T., Köhl, J. and Lambris, J.D., 2002, Essential role of the C5a receptor in E coli-induced oxidative burst and phagocytosis revealed by a novel lepirudin-based human whole blood model of inflammation. Blood 100, 1869-77.

Mollnes, T.E., Jokiranta, T.S., Truedsson, L., Nilsson, B., Rodriguez de Cordoba, S. and Kirschfink, M., 2007, Complement analysis in the 21st century. Mol Immunol 44, 3838-49.

Papp, K., Szekeres, Z., Erdei, A. and Prechl, J., 2008a, Two-dimensional immune profiles improve antigen microarray-based characterization of humoral immunity. Proteomics 8, 2840-8.

Papp, K., Szekeres, Z., Terényi, N., Isaák, A., Erdei, A. and Prechl, J., 2007, On-chip complement activation adds an extra dimension to antigen microarrays. Mol Cell Proteomics 6, 133-40.

Papp, K., Végh, P., Miklós, K., Németh, J., Rásky, K., Péterfy, F., Erdei, A. and Prechl, J., 2008b, Detection of complement activation on antigen microarrays generates functional antibody profiles and helps characterization of disease-associated changes of the antibody repertoire. J Immunol 181, 8162-9.

Prechl, J., Papp, K. and Erdei, A., 2010, Antigen microarrays: descriptive chemistry or functional immunomics? Trends Immunol 31, 133-7.

Reid, K.B., 1971, Complement fixation by the F(ab')2-fragment of pepsin-treated rabbit antibody. Immunology 20, 649-58.

Roumenina, L.T., Kantardjiev, A.A., Atanasov, B.P., Waters, P., Gadjeva, M., Reid, K.B., Mantovani, A., Kishore, U. and Kojouharova, M.S., 2005, Role of Ca2+ in the electrostatic stability and the functional activity of the globular domain of human C1q. Biochemistry 44, 14097-109. 
Seelen, M.A., Roos, A., Wieslander, J., Mollnes, T.E., Sjöholm, A.G., Wurzner, R., Loos, M., Tedesco, F., Sim, R.B., Garred, P., Alexopoulos, E., Turner, M.W. and Daha, M.R., 2005, Functional analysis of the classical, alternative, and MBL pathways of the complement system: standardization and validation of a simple ELISA. J Immunol Methods 296, 187-98.

Thielens, N.M., Cseh, S., Thiel, S., Vorup-Jensen, T., Rossi, V., Jensenius, J.C. and Arlaud, G.J., 2001, Interaction properties of human mannan-binding lectin (MBL)-associated serine proteases-1 and -2, MBL-associated protein 19, and MBL. J Immunol 166, 5068-77.

Trouw, L.A., Haisma, E.M., Levarht, E.W., van der Woude, D., Ioan-Facsinay, A., Daha, M.R., Huizinga, T.W. and Toes, R.E., 2009, Anti-cyclic citrullinated peptide antibodies from rheumatoid arthritis patients activate complement via both the classical and alternative pathways. Arthritis Rheum 60, 1923-31.

WACKER, W.E., IIDA, C. and FUWA, K., 1965, CONCENTRATION OF MAGNESIUM IN HUMAN PLASMA OR SERUM. Nature 206, 90.

Walport, M.J., 2001, Complement. First of two parts. N Engl J Med 344, 1058-66.

Weiler, J.M., Edens, R.E., Linhardt, R.J. and Kapelanski, D.P., 1992, Heparin and modified heparin inhibit complement activation in vivo. J Immunol 148, 3210-5.

Weiler, J.M. and Linhardt, R.J., 1989, Comparison of the activity of polyanions and polycations on the classical and alternative pathways of complement. Immunopharmacology 17, 65-72. 

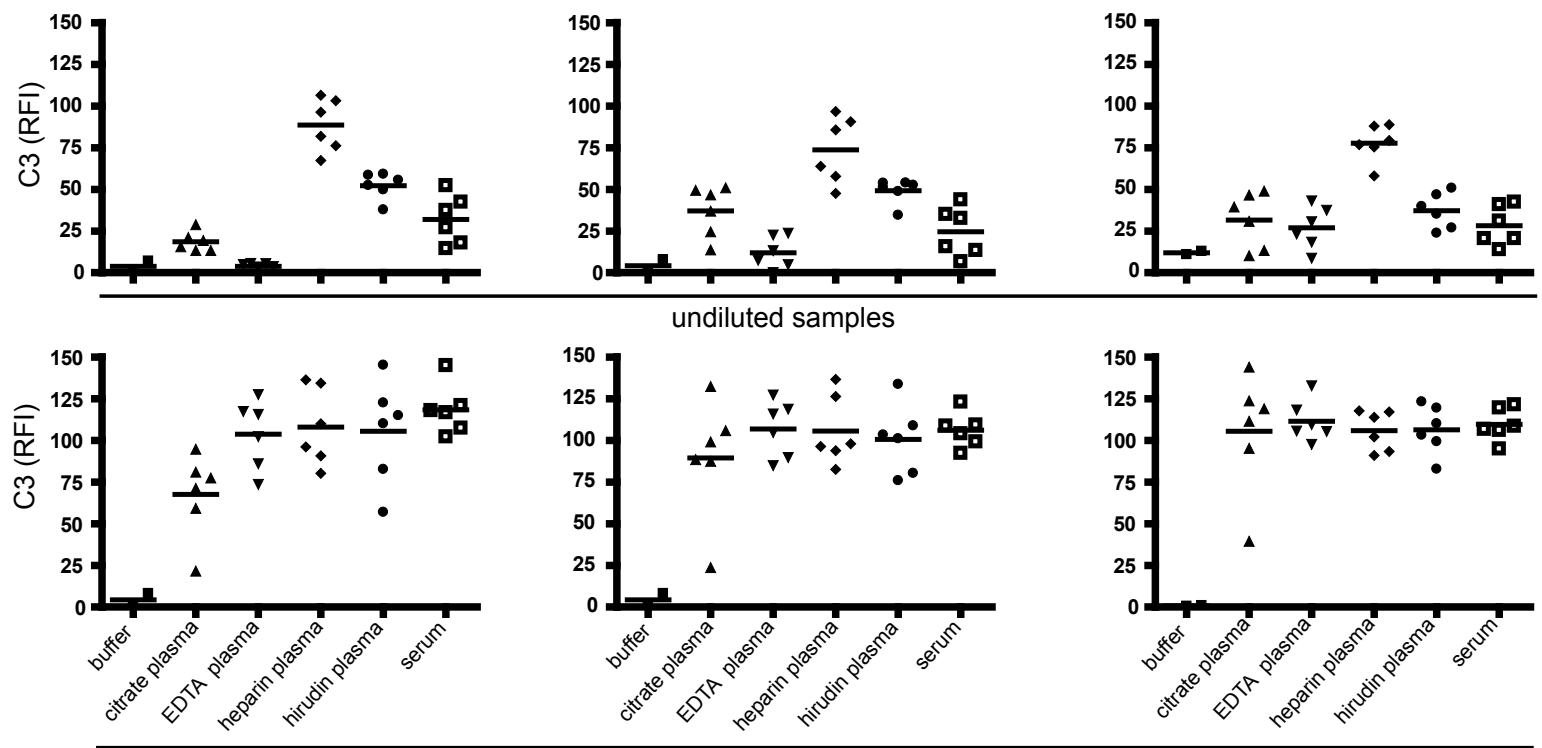

1:5 dilluted samples

B anti-human IgG

human IgG

properdin
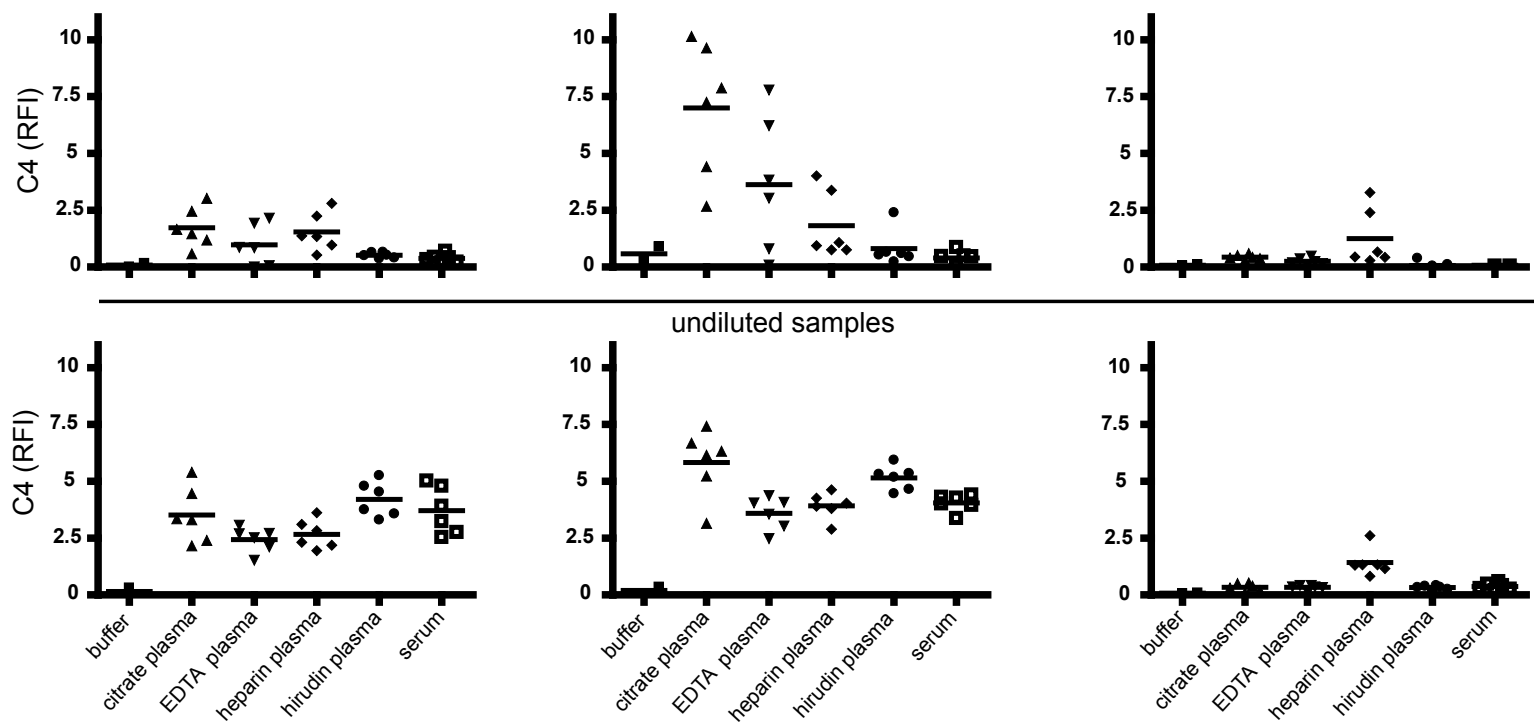

1:5 dilluted samples 
A
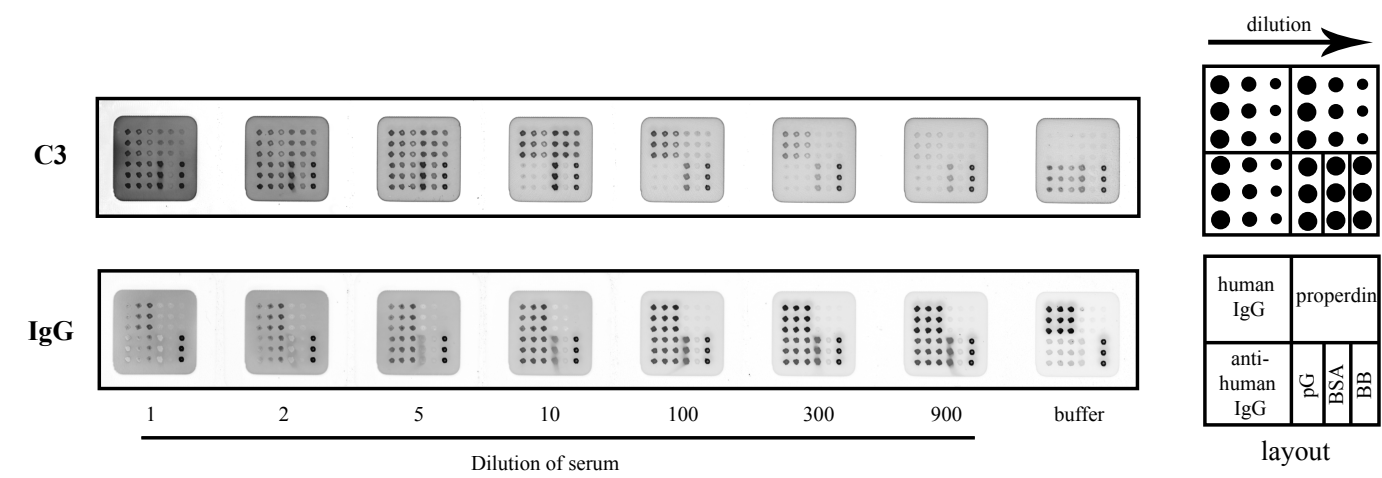

B
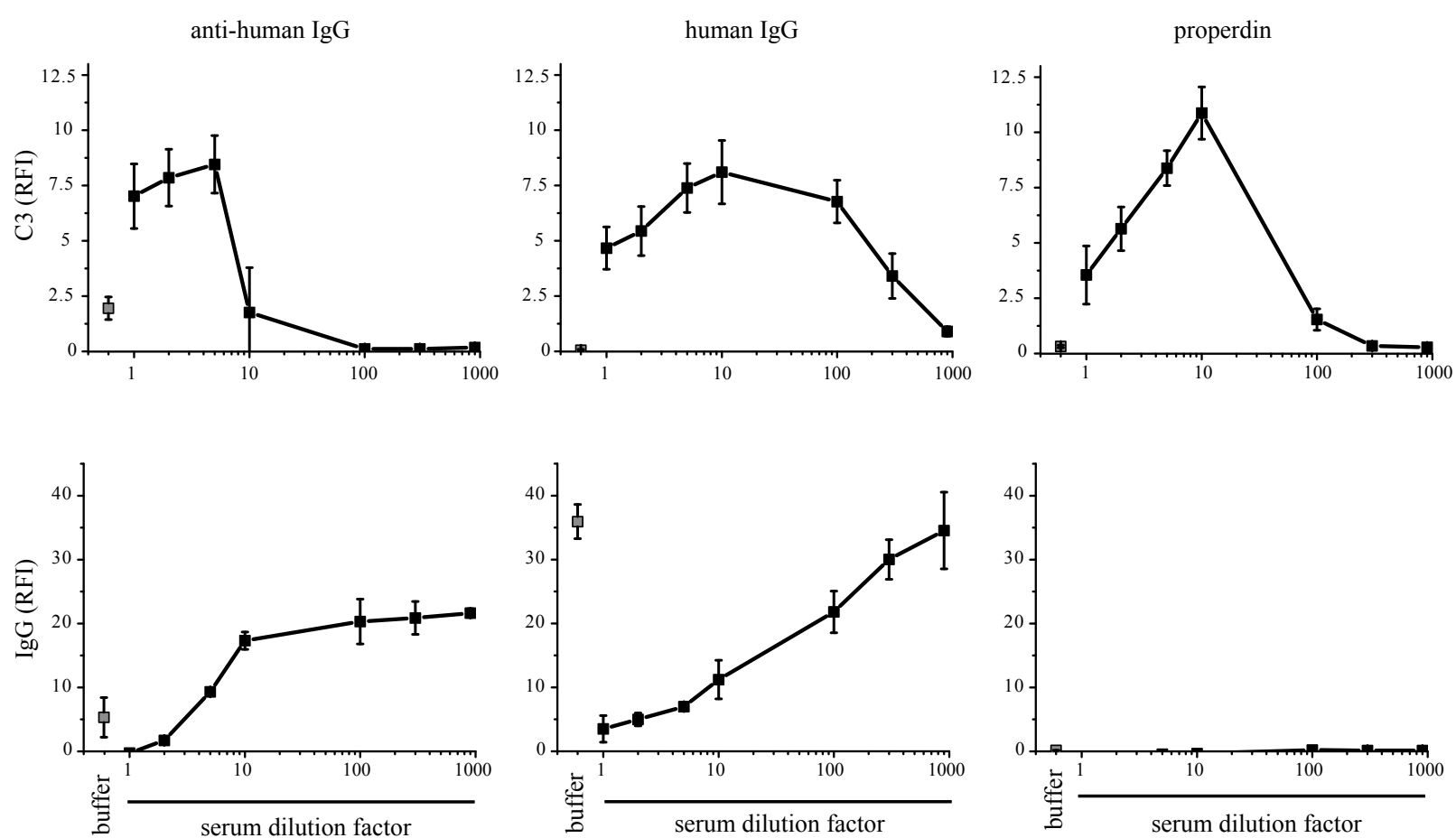

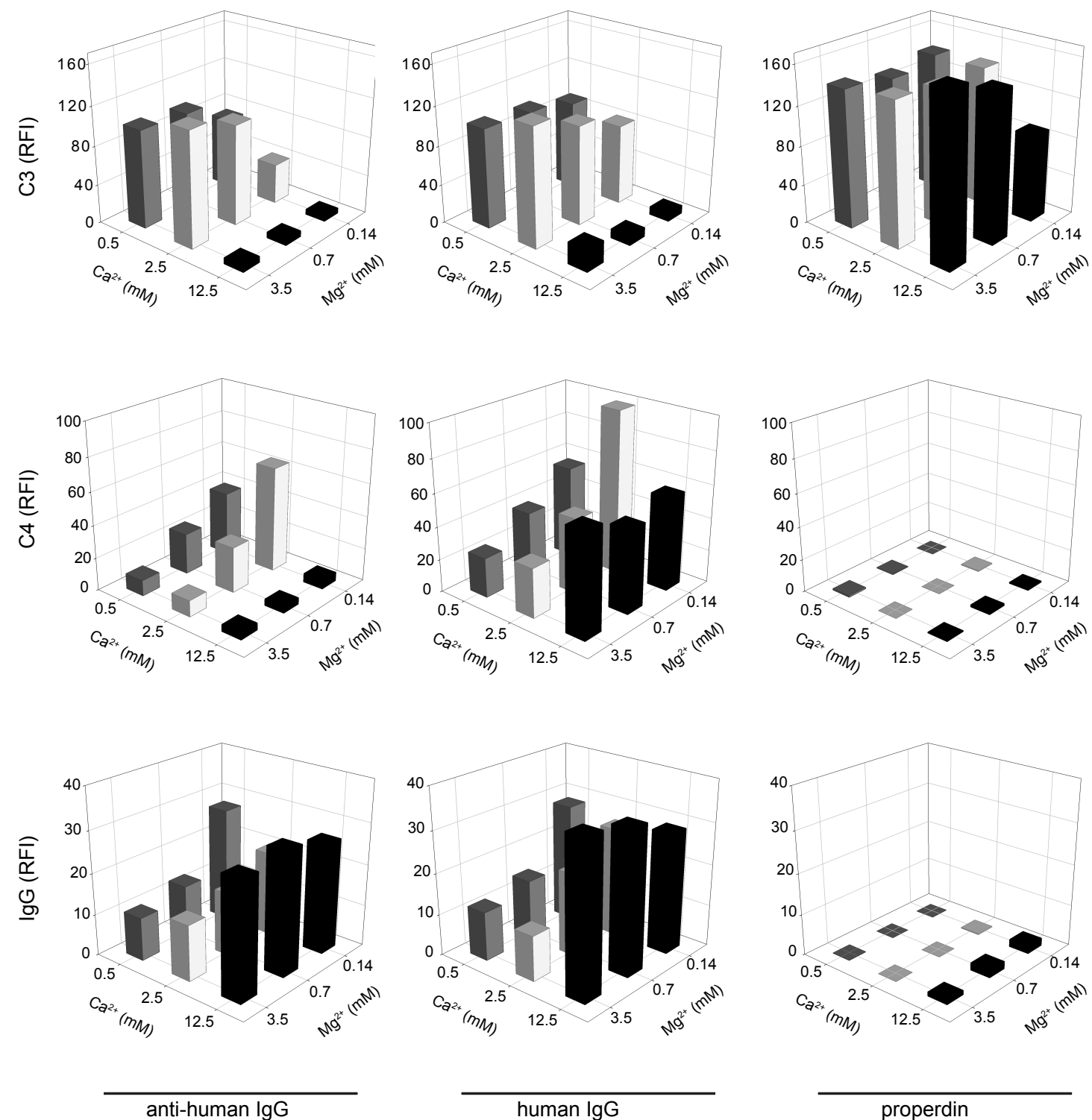

anti-human IgG

human IgG

properdin 


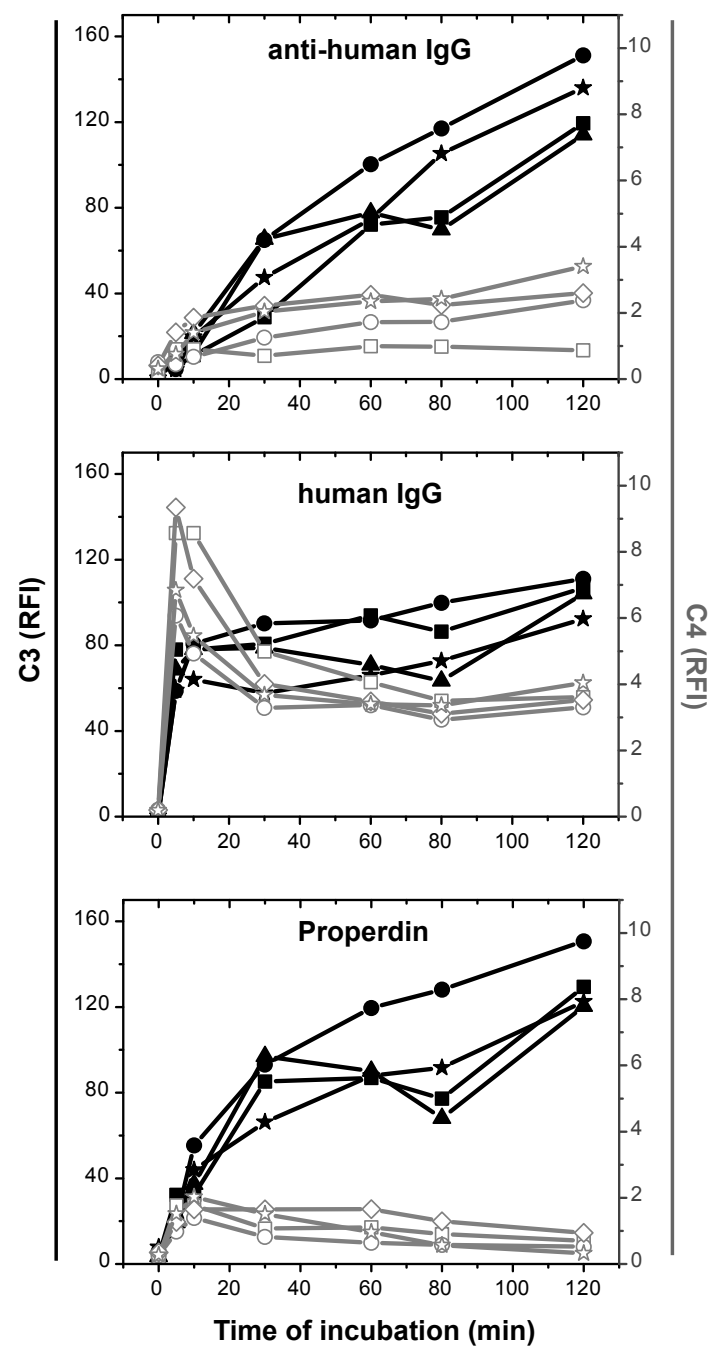




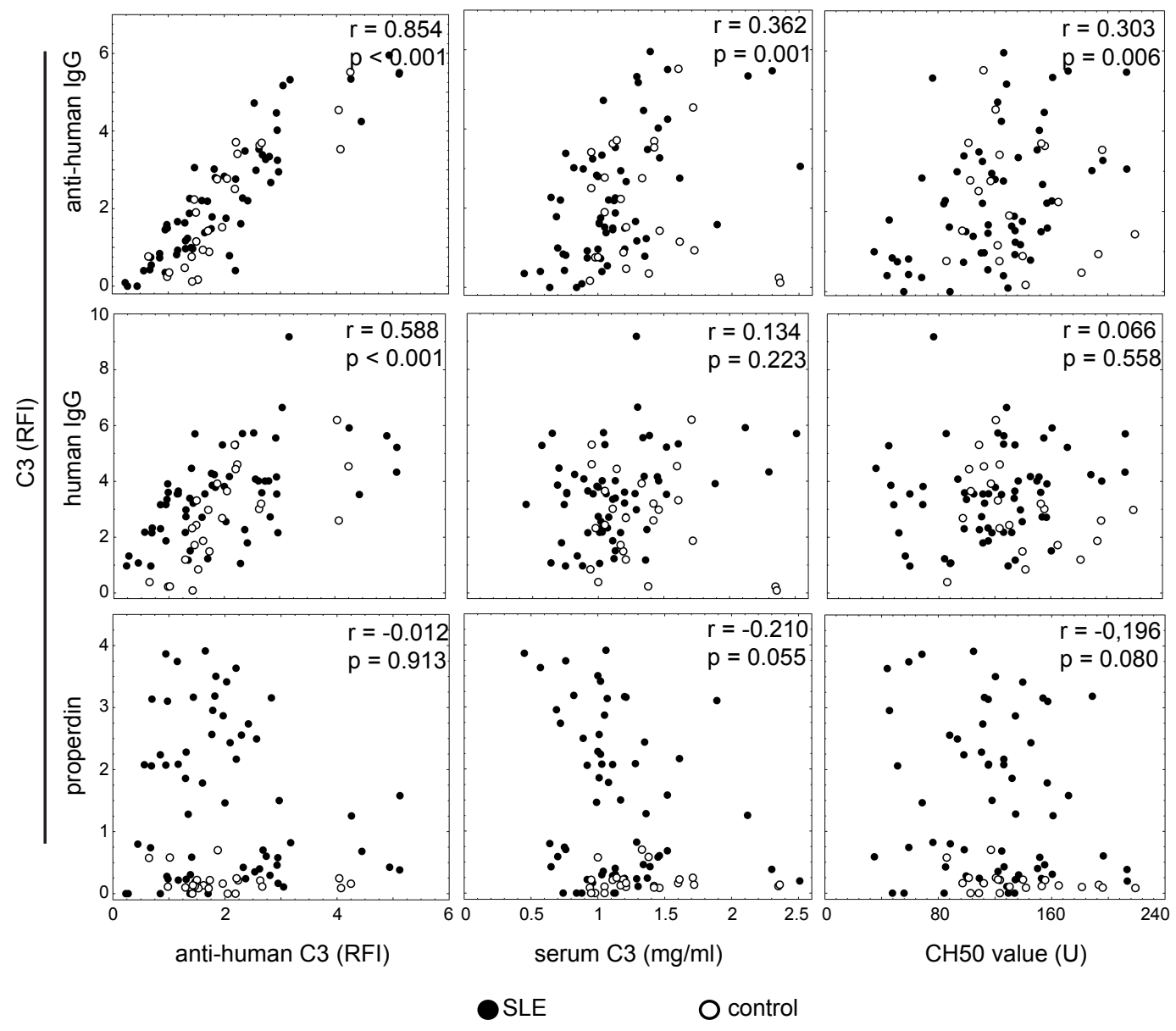

Figure 5. 